\title{
Actin, more than just a housekeeping protein at the scene of fertilization
}

\author{
Luigia SANTELLA* \& Jong T. CHUN \\ Stazione Zoologica Anton Dohrn, Napoli 80121, Italy
}

Received June 10, 2011; accepted June 20, 2011

\begin{abstract}
Since the first demonstration of sperm entry into the fertilized eggs of Mediterranean sea urchin Paracentrotus lividus by Hertwig (1876), enormous progress and insights have been made on this topic. However, the precise molecular mechanisms underlying fertilization are largely unknown. The two most dramatic changes taking place in the zygote immediately after fertilization are: (i) a sharp increase of intracellular $\mathrm{Ca}^{2+}$ that initiates at the sperm interaction site and traverses the egg cytoplasm as a wave, and (ii) the concomitant dynamic rearrangement of the actin cytoskeleton. Traditionally, this has been studied most extensively in the sea urchin eggs, but another echinoderm, starfish, whose eggs are much bigger and transparent, has facilitated experimental approaches using microinjection and fluorescent imaging methodologies. Thus in starfish, it has been shown that the sperm-induced $\mathrm{Ca}^{2+}$ increase in the fertilized egg can be recapitulated by several $\mathrm{Ca}^{2+}$-evoking second messengers, namely inositol 1,4,5-trisphosphate $\left(\mathrm{InsP}_{3}\right)$, cyclic ADP-ribose (cADPr) and nicotinic acid adenine dinucleotide phosphate (NAADP), which may play distinct roles in the generation and propagation of the $\mathrm{Ca}^{2+}$ waves. Interestingly, it has also been found that the dynamic rearrangement of the actin cytoskeleton in the fertilized eggs plays pivotal roles in guiding monospermic sperm entry and in the fine modulation of the intracellular $\mathrm{Ca}^{2+}$ signaling. As it is well known that $\mathrm{Ca}^{2+}$ regulates the structure of the actin cytoskeleton, our finding that $\mathrm{Ca}^{2+}$ signaling can be reciprocally affected by the state of the actin cytoskeleton raises an intriguing possibility that actin and $\mathrm{Ca}^{2+}$ signaling may form a 'positive feedback loop' that accelerates the downstream events of fertilization. Perturbation of the cortical actin networks also inhibits cortical granules exocytosis. Polymerizing actin bundles also compose the 'acrosome process,' a tubular structure protruding from the head of fertilizing sperm. Hence, actin, which is one of the most strictly conserved proteins in eukaryotes, modulates almost all major aspects of fertilization.
\end{abstract}

starfish, calcium, egg maturation, fertilization, PIP2, actin cytoskeleton, cofilin

Citation: $\quad$ Santella L, Chun J T. Actin, more than just a housekeeping protein at the scene of fertilization. Sci China Life Sci, 2011, 54: 733-743, doi: $10.1007 / \mathrm{s} 11427-011-4202-\mathrm{x}$

\section{Mutual activation of sperm and egg at ferti- lization}

To ensure successful fertilization and subsequent embryonic development, precisely controlled chain of events should take place both in the sperm and in the eggs at fertilization. The major part of our knowledge on the process of fertiliza-

*Corresponding author (email: santella@szn.it) tion has come from the studies on the invertebrate marine animals such as sea urchin and starfish that have served as fruitful experimental models for developmental biologists for more than a hundred years [1-3]. Fertilization of marine invertebrates usually takes place outside the body (external fertilization) and thus requires an extremely sensitive mechanism of chemotaxis between the eggs and the proper sperm. Recognition and interaction between the molecules on the sperm surface and the jelly layer surrounding the 
eggs thereby underlie the first step control of species-specific fertilization, which is followed by a series of physiological and morphological changes leading to acrosome (derived from Greek words Akros meaning 'topmost' and sōma meaning 'body') reaction on the sperm head. The acrosome reaction (AR) is basically a $\mathrm{Ca}^{2+}$-based exocytosis of the sperm's acrosomal vesicle, which contain digestive enzymes that pave the way for the fertilizing sperm. The species-specific egg jelly polysaccharides composed of fucose sulfate units, are known to trigger the AR in sea urchin [4]. In echinoderm, the head of sperm extends a long process (acrosomal process) filled with actin filaments. The surface of the acrosomal process of sea urchin sperm is covered with proteins called 'bindin' that mediate species-specific gamete adhesion and fusion by interacting with the highly glycosylated receptor molecules on the egg surface [5]. The external $\mathrm{Ca}^{2+}$ seems to play a pivotal role particularly in the acrosome reaction. Fertilization does not take place in $\mathrm{Ca}^{2+}$-free seawater as a result of failure of the acrosome reaction [6]. However, once exocytosis of the acrosomal vesicle is induced by treatment of the sperm with the egg jelly in $\mathrm{Ca}^{2+}$-containing seawater, the subsequent fertilization steps can take place successfully even in $\mathrm{Ca}^{2+}$-free seawater [7].

The first detailed analysis of the AR in marine animals has been made by Dan et al [8]. She showed the effects of egg jelly-containing seawater on starfish spermatozoa. The first visible effects were: (i) the formation of sperm clusters as a result of the sperm heads' agglutination, (ii) the exocytosis of the acrosomal vesicle in the sperm, (iii) the fast extension of a long (ca. $25 \mu \mathrm{m}$ ) and rigid acrosomal process, and (iv) the rearrangement of the principal part of the reacted sperm. During the AR in sea urchin sperm, a rapid (within 15 s) and continuous uptake of ${ }^{45} \mathrm{Ca}$ occurred following the exposure to the egg jelly [9]. According to the X-ray microanalysis of frozen sea urchin sperm that had been activated by the egg jelly, a large increase in calcium and sodium takes place in the mitochondria during the acrosome reaction [10]. In triggering the $\mathrm{AR}$ in starfish sperm, three components of egg jelly are known to cooperate with one another: namely, ARIS (AR-inducing substance), co-ARIS, and asterosap. For example, in sea urchin and starfish, asterosap works as a chemotactic agent and acts on a receptor associated with guanylyl cyclase located in the sperm flagellar plasma membrane and thereby stimulates the motility of the sperm. This is achieved through the rapid intracellular synthesis of cGMP (the level increases up to 60-fold within 100-200 ms), the increase of $\mathrm{pH}$, and the transient elevation of $\mathrm{Ca}^{2+}$ caused by a $\mathrm{Na}^{+} / \mathrm{Ca}^{2+}$ exchanger $[11,12]$.

\section{Electrical events at the plasma membrane during sperm-egg fusion}

The response of the egg to the sperm also involves a transi- ent shift of the membrane potential from negative to positive values, which eventually returns to the negative levels about $10 \mathrm{~min}$ after the insemination [13]. Three distinct fertilization-linked electrical events have been characterized in sea urchin eggs. The first is a minute step-like plasma membrane depolarization of 1-2 $\mathrm{mV}$ in amplitude. This 'latent period' reflects the initial sperm-egg fusion and is also characterized by a concomitant decrease in electric resistance of the egg plasma membrane by the induction of the sperm ion channels. Then follows a steep depolarization of the membrane potential that leads to a regenerative action potential, and the membrane potential remains near or above $0 \mathrm{mV}$ (activation potential) due to the sodium and calcium influx [14-16].

Studies on the block to polyspermy in sea urchin led to the conclusion that a fast reaction transmits over the surface of the egg in about two seconds which reduces the chances of re-fertilization by a factor of 20 [17]. This fast reaction is followed by a slower (about 60 seconds) change that reduces to zero the probability of a successful sperm-egg collision. Jaffe [13] suggested that in Strongylocentrotus purpuratus the initial positive membrane potential which is attained in about 3 seconds after a successful sperm attachment reduces the probability of supernumerary sperm fusion. It was also demonstrated that the artificial depolarization of eggs prevented egg activation and sperm entry. However, the hypothesis of the electrical fast block to polyspermy has not been universally accepted (reviewed in $[18,19]$ ) since no evidence for a fast block was obtained in $S$. purpuratus by others [20]. Indeed, in sea urchin eggs whose interaction with sperm was quickly interrupted within 20 seconds after insemination by a brief exposure to warm seawater containing detergent, sperm can re-fertilize and freely enter the eggs at the unfertilized zones, indicating that block to polyspermy is more linked to the local changes in the egg cortex (cortical reaction) [21]. Similarly, partially fertilized sea urchin eggs whose cortical reaction was interrupted by heat shock gave rise to multiple step-depolarizations that probably resulted from several sperm fusing with the "inactivated" egg surface [22]. Finally, arguing against the view of the membrane potential-based fast block to polyspermy, configuration of voltage clamping to hold the sea urchin eggs at negative membrane potential produced monospermic fertilization, and not polyspermy (reviewed in [19]).

Immature oocytes of sea urchin eggs do not undergo cortical granule exocytosis at fertilization and are more prone to polyspermy than the mature eggs. Physiological and morphological studies have shown that immature oocytes of sea urchin are more receptive to spermatozoa than the mature eggs, as they exhibit the first electrical event as early as 5 seconds after insemination, compared to about 13 seconds for the mature eggs. No further changes occur until $50 \mathrm{sec}$ later, when the spermatozoon stops gyrating, and the oocyte surface swells up at the point of sperm attachment to engulf the spermatozoon. Not having cortical granule exocytosis, 
10-15 spermatozoa enter the oocytes as judged by the input resistance decaying progressively according to the number of sperm entries, although the first sperm usually induces activation potential of $50 \mathrm{mV}$ in amplitude [23]. These results again display the importance of cortical granule exocytosis in blocking polyspermy and suggest that a limited number of successful interaction sites on the oocyte surface become less receptive during maturation.

\section{The cortical granules exocytosis and the block to polyspermy}

At fertilization, a series of changes take place in the egg surface. This 'cortical reaction' begins at the sperm attachment site and lasts no longer than two minutes in sea urchin. Nonetheless, it alters the fate of the egg, as the unfertilized eggs would eventually die within hours. Beneath the thick layer of the jelly, the surface of the unfertilized egg is covered with the vitelline layer which can be digested away with trypsin or chymotrypsin [24]. At fertilization, this layer is detached from the plasma membrane, creating 'perivitelline space.' Hydration of the exudates of cortical granules such as mucopolysaccarides and the enzymatic activity of proteases promote dispersal of the released granule contents into the perivitelline space, and the process transforms the vitelline layer into the 'fertilization envelope' that naturally lifts off the unfertilizing spermatozoa and thereby serves as an ultimate block to polyspermy [25]. On the other hand, at the plasma membrane side of the perivitelline space, a clear layer is being formed as a result of cortical granule exocytosis, which becomes hardened by excess calcium and softened by the lack of it. Hyaline is its major protein of this layer, as it was isolated and characterized as a calciuminsoluble material that is released after the fertilization [26].

The cortical granules (secretory vesicles beneath the egg plasma membrane) were first described by Motomura [27] who stained them in frog eggs with 'Janus green' before and after fertilization. He found that the surface granules in the egg of Arbacia, which could not be dislocated even by centrifugation, had readily disappeared after fertilization. Before fertilization or artificial activation, the cortical granules are firmly anchored to the cortical layer, but the fertilization impulse weakens the rigidity (stiffness) of the cortex and triggers exocytosis of the granules. The subsequent membrane elevation is evident even under the light microscope [28-31], and the wave of the fusion between the membranes of individual cortical granules and the overlying plasma membrane starts from the sperm-egg interaction site and propagates to the opposite side of the egg. The excess membrane of cortical granules fused to the plasma membrane is in part accommodated by the elongating microvilli, whereas the reminder undergoes a rapid and massive endocytotic re-absorption into the cytoplasm [25]. Within 10-30 min post-insemination, the soft fertilization envelope of the zygote gradually hardens owing in part to a series of covalent cross-linking activity of cortical granule-derived peroxidases using $\mathrm{H}_{2} \mathrm{O}_{2}$ that is produced by the fertilized egg, and to the $\mathrm{Ca}^{2+}$ and $\mathrm{Na}^{+}$-dependent structural modifications [32,33].

\section{Calcium signaling at fertilization}

It is well known that at fertilization the spermatozoon activates the egg by triggering a massive release of $\mathrm{Ca}^{2+}$ that starts at the point of sperm interaction and traverses the egg cytoplasm as a wave. Depending on the species, the $\mathrm{Ca}^{2+}$ increase may occur as a single transient or as repetitive oscillations [2]. At least for the eggs of invertebrates, however, there is no consensus on the mechanisms of the release, i.e. on the upstream signaling pathway and the nature of the intracellular $\mathrm{Ca}^{2+}$ stores. Three major hypotheses have emerged on the mechanism of egg activation. Firstly, the sperm may bind to the receptor on the egg plasma membrane, which mediates the signal transduction through the G-protein/PLC/InsP $\mathrm{P}_{3}$ pathway. This hypothesis is supported by the analysis of the reaction time for the breakdown of cortical granules in sea urchin eggs upon microinjection of inositol 1,4,5-trisphosphate $\left(\mathrm{InsP}_{3}\right)$ and by the use of the hydrolysis-resistant analog of GTP guanosine-5'-0-(3-thiotriphosphate) (GTP $\gamma \mathrm{S})$, or by the use of GDP guanosine-5'-0-(2-thiodiphosphate) (GDP $\beta S$ ) which prevented sperm from stimulating exocytosis [34,35]. However, this suggestion was not substantiated, as GDP $\beta S$ at high concentration could have a non-specific inhibitory action [36]. As a slightly modified model, a receptor tyrosine kinase pathway involving the activation of $\gamma$ isoform of the PLC and $\mathrm{InsP}_{3}$ formation was also proposed [37]. Secondly, the sperm may infuse a diffusible factor into the egg. This 'sperm factor hypothesis' is based on the finding that microinjection of a cytosolic extract from spermatozoa caused the cortical granules exocytosis and $\mathrm{Ca}^{2+}$ oscillations [38,39] and that injection of sperm extract in ascidian oocytes induced a membrane current similar to that promoted by the sperm itself [40]. Initially, the putative $\mathrm{Ca}^{2+}$-releasing sperm factor has been proposed to be a second messenger such as $\mathrm{InsP}_{3}$ and NAADP, or a soluble protein such as oscillin that would diffuse into the egg cytoplasm after gamete fusion [41-43]. More recently, a novel type of sperm-specific PLC, PLC $\zeta$, has been proposed as the sperm factor since microinjection of cRNA encoding the sperm PLC $\zeta$ caused fertilization-like $\mathrm{Ca}^{2+}$ oscillations in mouse eggs [44]. Finally, the third model is based on the 'sperm conduit hypothesis' according to which $\mathrm{Ca}^{2+}$ itself would enter the egg through the acrosomal process $[45,46]$. The hypothesis relied on the calculations of the high rates of ${ }^{45} \mathrm{Ca}^{2+}$ entry into sea urchin sperm measured both during and after the acrosome reaction [9].

In echinoderm and vertebrate eggs, the rise of cytosolic 
$\mathrm{Ca}^{2+}$ at fertilization has been attributed, in large part, to the $\mathrm{Ca}^{2+}$ release from the endoplasmic reticulum (ER) in response to the synthesis of $\mathrm{InsP}_{3}$ (reviewed in [3,47-49]). In starfish, $\mathrm{Ca}^{2+}$ signaling at fertilization may involve a subtype of PLC utilizing Src-like protein tyrosine kinase (PLC $\gamma$ ) rather than the one activated by G-proteins (PLC $\beta$ ). Recombinant proteins containing the two SH2 domains of PLC $\gamma$ that specifically inhibit PLC $\gamma$ but not PLC $\beta$ completely blocked $\mathrm{Ca}^{2+}$ release at fertilization, suggesting that the $\mathrm{Ca}^{2+}$ release was due to the activation of PLC $\gamma$ and $\mathrm{InsP}_{3}$ formation [50]. Subsequent experiments indicated requirement of a sequential activation of Src family kinase and PLC $\gamma$ for initiating $\mathrm{Ca}^{2+}$ release in starfish and sea urchin eggs at fertilization [51-53]. In further support of ER as a reservoir of $\mathrm{Ca}^{2+}$ at fertilization, mature Xenopus eggs displayed specialized plasma membrane-ER junctions that are structurally analogous to the junctions between the plasma membrane and the sarcoplasmic reticulum in the muscle [54]. The polarized distribution of the egg plasma membrane-ER junctions along the animal-vegetal axis could be responsible for the greater excitability and the propagation speed of the $\mathrm{Ca}^{2+}$ wave at the animal hemisphere [55].

\section{The role of $\mathrm{Ca}^{2+}$ in egg activation and cortical granules exocytosis}

The role of $\mathrm{Ca}^{2+}$ in triggering activation of quiescent eggs and the cortical reaction was first proposed by Heilbrunn [56] and by his student Mazia [57] who showed that "a primary effect of a stimulating agent on a cell is to cause a release of calcium from organic combinations in the cortex of the cell into the main body of protoplasma." In this work, suspensions of unfertilized and fertilized Arbacia eggs were used to quantify the amount of bound and free $\mathrm{Ca}^{2+}$ before and after fertilization. Direct measurement of the intracellular $\mathrm{Ca}^{2+}$ increase upon fertilization was later provided by Ridgway et al [58] who showed that the wave of membrane elevation in fertilized eggs of medaka was preceded by a wave of cortical granule exocytosis. A massive release of free cytosolic $\mathrm{Ca}^{2+}$ was visualized with aequorin in the fertilized eggs of Medaka eggs. The $\mathrm{Ca}^{2+}$ wave and the egg activation started from the sperm-egg interaction site and traveled to the opposite side, leading to the idea that an alteration in the intracellular concentration of $\mathrm{Ca}^{2+}$ was the signal that initiates the development of the fertilized egg.

Evidence that $\mathrm{Ca}^{2+}$ liberated from an intracellular compartment is essential for the breakdown (exocytosis) of cortical granules was provided by Steinhardt and coworkers [59] who showed that the $\mathrm{Ca}^{2+}$ ionophore A23187 triggered the cortical reaction in the absence of external $\mathrm{Ca}^{2+}$. Indeed, the addition of $\mathrm{Ca}^{2+}$ to cortical granules isolated from sea urchin eggs using a procedure retaining their physiological activity induced the swelling of individual granules and their lateral fusion during cortical granule breakdown, indicating a self-propagating nature of the cortical reaction by the release of $\mathrm{Ca}^{2+}$ from the local stores $[60,61]$. Studies on the ultrastructure of the eggs at exocytosis showed that a single layer of cortical granules is tightly attached to the plasma membrane in the egg cortex through a network of fine filaments [62-64].

\section{Meiotic maturation and fertilization of star- fish eggs}

The fully grown oocytes of starfish are arrested at the first prophase of meiosis within the gonad until the time of spawning at sea. The starfish oocyte of this stage is characterized by the presence of the clearly delineated nucleus termed 'germinal vesicle (GV)' (Figure 1). In the laboratory, immature oocytes of starfish can be easily matured with the use of 1-methyladenine (1-MA) that stimulates resumption of the meiotic cell cycle. Hence, at variance with sea urchin, starfish provides an optimal experimental opportunity to study both meiotic maturation and fertilization [65]. The maturation process includes the dissolution of the nuclear envelope (GVBD, germinal vesicle breakdown) and the two rounds of polar body formations that are extruded on a regular time schedule. During meiotic maturation, oocytes undergo physiological and morphological changes in preparation for fertilization, and as mentioned above, the capacity for cortical granules exocytosis and for the block to polyspermy are fully functional only in the mature eggs. Thus, the period for an optimal fertilization ensuring successful egg activation and subsequent development is between GVBD and the extrusion of the first polar body in starfish [66].

During the maturation process, the mechanical properties of starfish oocytes also change. After 1-MA treatment, surface tension of the oocyte decreases rapidly and remains low until the extrusion of the first polar body. A sharp rise and fall in tension is again observed during formation of the second polar body. The cyclic activity of cortical tension appears to be the result of the intermixture of nucleoplasm and cytoplasm, as it occurs after the dissolution of the nuclear envelope, and not in the fragmented oocytes lacking the nucleus $[67,68]$. During maturation, the electric property of the plasma membrane also changes. In starfish oocyte, the initial resting potential marks $-80 \mathrm{mV}$ but shifts to a new stable level of -10 to $-20 \mathrm{mV}$ in response to $1-\mathrm{MA}[69,70]$.

In parallel to this, the ultrastructure of the oocytes undergoes significant changes during meiotic maturation. Starfish oocytes not treated with 1-MA contain relatively long microvilli emanating from the cell surface into the vitelline layer. In these oocytes, cortical granules characteristically charged with electron dense components are scattered along the periphery of the cortex [71]. Upon the addition of 1-MA, however, a rapid rearrangement of the oocyte 

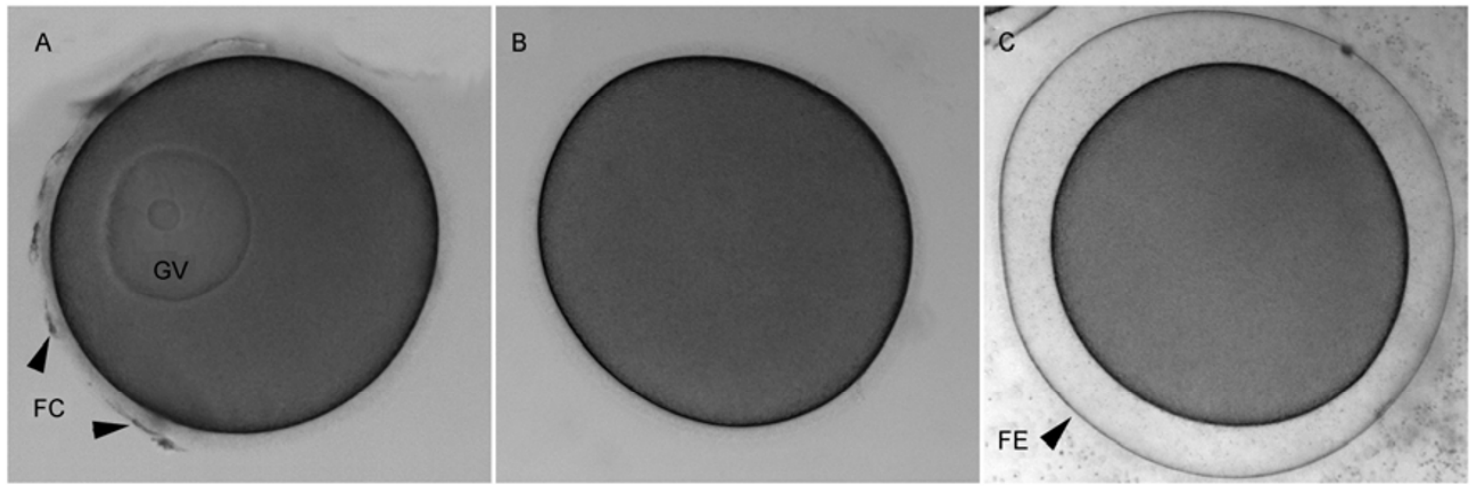

Figure 1 Maturation and fertilization of starfish eggs. A, Fully grown immature oocytes of Astropecten aranciacus are arrested at the first prophase of meiotic division, containing a large nucleus (termed germinal vesicle, GV). The follicle cells (FC) surround the outer layer of the egg jelly coat. B, The same oocyte treated for $1 \mathrm{hr}$ with the maturing hormone 1-methyladenine (1-MA). The nuclear envelope is disassembled and the cytoplasm is mixed with the nucleoplasm. C, A fertilized egg with the fertilization envelope (FE) formed as a result of the cortical granules exocytosis.

surface ensues within a few minutes. On the egg surface, actin is polymerized and subsequently depolymerized as the actin spikes arise and disappear [72]. Microvilli diminish their length following GVBD, and the cortical granules move to the subjacent areas of the plasma membrane forming a monolayer. They orient themselves with their long axis perpendicular to the plasma membrane, a process dependent on the actin cytoskeleton [73]. In addition to actin, changes in other cytoskeletal components such as microtubules and the intermediate filaments have been described in the maturing oocytes $[74,75]$.

Concomitant with the cytoskeletal changes, the endoplasmic reticulum (ER) is known to undergo structural and functional changes during the maturation. In several animal species, the structure of the ER of the maturing oocytes appears more clustered in the cortical and subcortical regions in comparison with the oocytes at the GV stage [76]. In starfish oocytes, microinjection of an oil drop saturated with a fluorescent lipophilic dye showed profound structural changes in the ER membranes during the 1-MA-induced maturation [77]. This structural transition of the ER seems to be closely lined to the increased clustering of $\mathrm{InsP}_{3}$ receptors $\left(\mathrm{InsP}_{3} \mathrm{R}\right)$ that was observed during egg maturation. As the $\mathrm{Ca}^{2+}$-induced $\mathrm{Ca}^{2+}$ release at the activation phase of Ins $\mathrm{P}_{3} \mathrm{R}$ would be much facilitated by densely packed $\operatorname{Ins} \mathrm{P}_{3} \mathrm{R}$, these ER-associated changes of $\operatorname{InsP}_{3} \mathrm{R}$ distribution may contribute to the optimized $\mathrm{Ca}^{2+}$ response at fertilization [76,78]. Besides maturation-induced ER remodeling, activity of the cell cycle-related molecules such as MPF (M-phase promoting factor) may fine-tune the biochemical response of the eggs [76]. In Xenopus, the $\mathrm{InsP}_{3}$-induced $\mathrm{Ca}^{2+}$ response is enhanced at the GVBD stage, and the process is correlated with MPF activation. Three residues, Thr-931, Thr-1136, and Ser-114, of the $\operatorname{Ins}_{3} \mathrm{R}$ are specifically phosphorylated during maturation, and the phosphorylation of Thr-1136 requires MPF activation [76,79]. The recent finding that the mitogen-activated protein kinase (MAPK) cascade is also important for shaping $\mathrm{Ca}^{2+}$ dynam- ics in mouse eggs [80] adds more weight to the suggestion that phosphorylation plays an important role in the sensitization of $\mathrm{InsP}_{3}$-dependent $\mathrm{Ca}^{2+}$ release.

\section{Calcium signaling during maturation and fertilization of starfish eggs: focus on the actin cytoskeleton}

\section{1 $\mathrm{Ca}^{2+}$ signaling during oocyte maturation}

The importance of $\mathrm{Ca}^{2+}$ in triggering meiosis re-initiation in starfish oocytes has been the subject of vigorous debates [81]. The binding of 1-MA to cell surface receptors triggers a transient increase of intracellular $\mathrm{Ca}^{2+}$ within few minutes both in the intact oocytes and in the isolated cortices of starfish oocytes [82]. The direct role of the $\mathrm{Ca}^{2+}$ signaling in resumption of meiosis was questioned by the finding that buffering of cytoplasmic $\mathrm{Ca}^{2+}$ by injecting EGTA or BAPTA did not inhibit meiosis resumption [83]. However, Santella and coworkers [84] found that 1-MA induces $\mathrm{Ca}^{2+}$ elevation in the nucleus following its increase in the cytoplasm. This nuclear increase of $\mathrm{Ca}^{2+}$ is required for GVBD in the starfish Asterina pectinifera since the injection of BAPTA directly into the nucleus prevented the dissolution of the nuclear envelope in the oocytes treated with 1-MA [84,85]. More recently, it was demonstrated that the 1-MA-mediated $\mathrm{Ca}^{2+}$ increase always initiates at the vegetal hemisphere of the oocytes and that the sites of $\mathrm{Ca}^{2+}$ release are largely restricted to the subplasmalemmal regions. The $\mathrm{Ca}^{2+}$ wave arrives at the opposite side of the oocytes in about 20 seconds [86]. Apparently, the $\mathrm{Ca}^{2+}$ liberation by 1-MA was not linked to the second messengers, namely $\mathrm{InsP}_{3}$, cADPr or NAADP, but was inhibited by the structural alteration of the cortical actin cytoskeleton. In line with this finding, all the tested agents that promote assembly or disassembly of the actin cytoskeleton e.g. latrunculinA (LAT-A), heparin, G-proteins inhibitors, and jasplakinolide (Figure 2) significantly affected the spatiotemporal pattern of 
the 1-MA-induced $\mathrm{Ca}^{2+}$ release [86,87]. Furthermore, the 1-MA-induced cortical $\mathrm{Ca}^{2+}$ release was also potentiated by the actin binding protein cofilin that reorganizes the actin cytoskeleton, as its preinjection into the starfish oocytes enhanced the $\mathrm{Ca}^{2+}$ liberation nearly by two-fold [88].

During meiotic maturation, starfish oocytes become more sensitive to the stimuli that induce an increase of intracellular $\mathrm{Ca}^{2+}$, e.g. sperm, InsP 3 , cADPr and NAADP [89-91]. Thus, in comparison with immature oocytes, mature eggs of starfish release higher level of $\mathrm{Ca}^{2+}$ in response to the same amount of $\mathrm{InsP}_{3}$. Indeed, the longer the oocytes are exposed to 1-MA, the higher level of $\mathrm{Ca}^{2+}$ is released [92]. Interestingly, this apparent sensitization to $\mathrm{InsP}_{3}$ is negated by the pretreatment of the oocytes with the actin-depolymerizing agent LAT-A, suggesting that the actin cytoskeleton is implicated in the optimization of the intracellular $\mathrm{Ca}^{2+}$ release mechanism [92]. In line with the idea, the actin cytoskeleton is dynamically rearranged in response to 1-MA concomitantly with or slightly before the ER reorganization [77] (Figure 3). In response to 1-MA, the GVBD-stage oocytes begin to be sensitized to $\operatorname{InsP}_{3}$ starting from the perinuclear area near the animal pole and the effect spreads to the entire oocyte along the animal-vegetal axis (Figure 4). The increased sensitivity in the perinuclear area was delayed by about $30 \mathrm{~min}$ in oocytes preincubated with roscovitine, a specific inhibitor of MPF [92]. At variance with other animal species [93], however, MPF does not seem to directly phosphorylate the $\mathrm{InsP}_{3}$ receptors in starfish oocytes [92,94]. Hence, the exact mechanism by which actin contributes to the sensitization of the intracellular $\mathrm{Ca}^{2+}$ releasing system is yet to be resolved. In this regard, it is noteworthy that LAT-A by itself induces both $\mathrm{Ca}^{2+}$ influx and the release from the intracellular stores in the mature eggs of $A$. aranciacus after 10 minutes incubation [95]. This massive increase of intracellular $\mathrm{Ca}^{2+}$ by LAT-A parallels the progress of actin depolymerization that continues nearly for $1 \mathrm{hr}$, but the phenomenon is not observed in immature oocytes. Thus, the effect may be linked to the egg's sensitization of the $\mathrm{Ca}^{2+}$ release mechanism such as ion channels, or may involve a novel mechanism that directly involves actin treadmilling [92].

\section{2 $\mathrm{Ca}^{2+}$ signaling during egg fertilization}

Owing to the big cell size (up to $170 \mu \mathrm{m}$ in diameter) and the optical transparency of the cytoplasm, starfish eggs have served as a useful model for studying the sperm-egg interaction, $\mathrm{Ca}^{2+}$ signaling and sperm incorporation during fertilization. The sperm acrosome reaction occurs when the spermatozoon makes contact with the outer layer of the jelly coat. Being connected to the egg by a long acrosomal process, the fertilizing spermatozoon of starfish activates the egg when it is quite at a distance from the egg's plasma membrane. This unique mode of initial contact between the

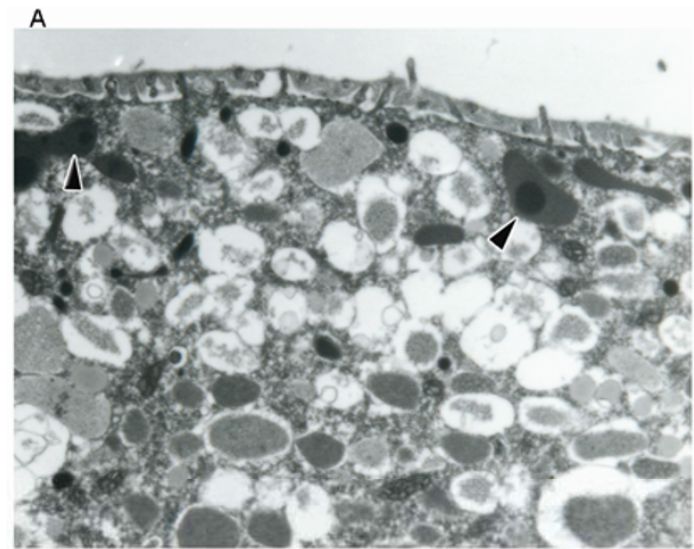

B
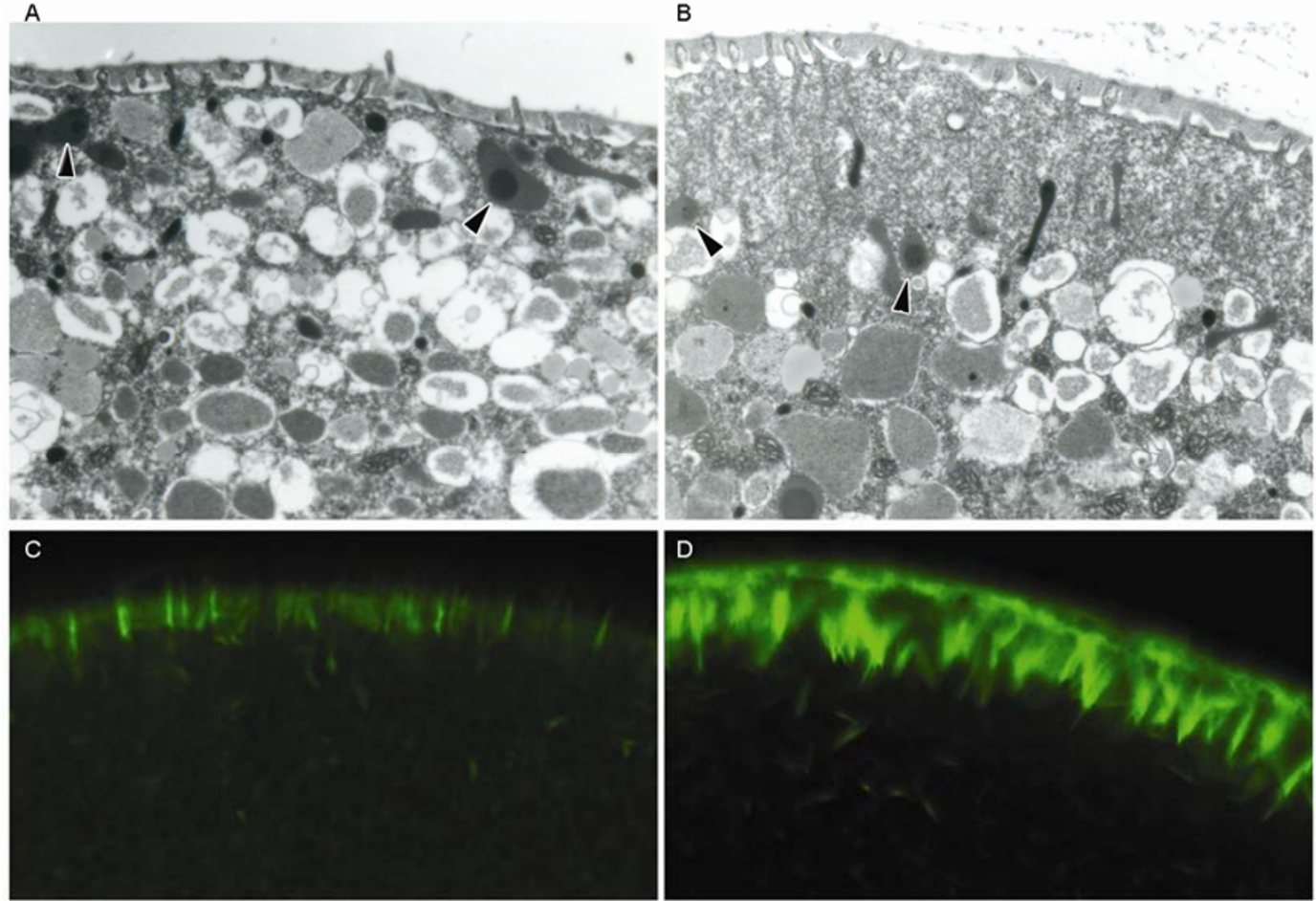

Figure 2 Structural changes in the cortex of immature oocytes. A, Ultrastructural analysis of the cortex of an immature oocyte observed in the transmission electron microscope. The cortical granules (arrows) are visible beneath the plasma membrane. B, Changes in the structural organization of the oocyte cortex following jasplakinolide treatment (final concentration $12 \mu \mathrm{mol} \mathrm{L} \mathrm{L}^{-1}$ ). The cortical actin polymerization dislodges the cortical granules (arrows) and the vesicles away from the plasma membrane. C, F-actin staining in a living immature control oocyte by the microinjection of Alexa Fluor 488-conjugated phalloidin. D, Enhancement of the cortical actin networks by the same incubation with jasplakinolide. 

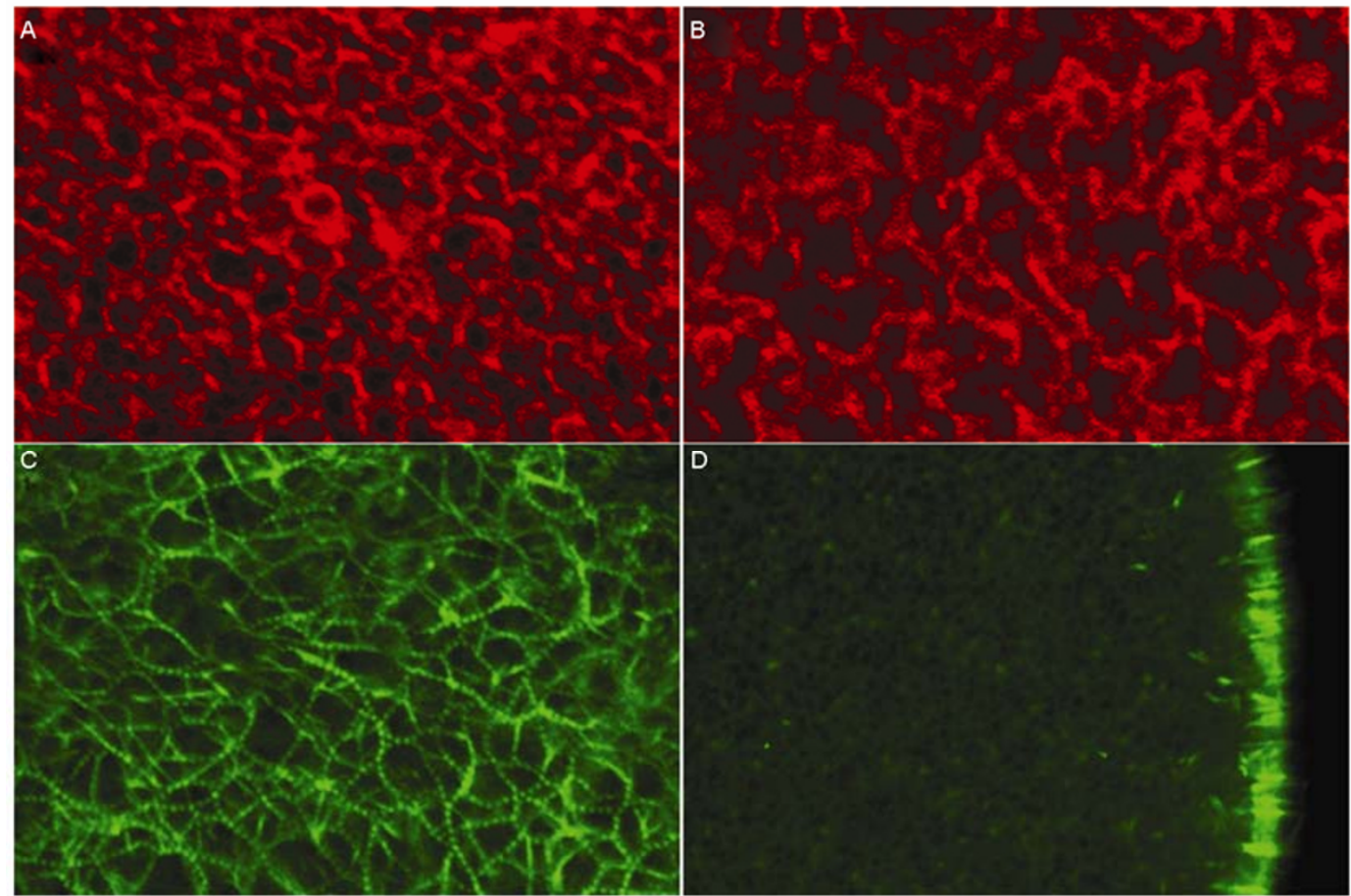

Figure 3 Structural changes of the ER and the actin cytoskeleton during the egg maturation of starfish. A, Confocal image of ER stained with the oil droplet saturated with a lipophilic dye in an immature oocyte. B, Changes in the ER morphology following the maturation by 1-MA. C, Actin filaments in a living immature oocyte visualized with injected Alexa Fluor 488-conjugated phalloidin. D, The actin cytoskeleton network is no longer visible in a mature egg treated for $1 \mathrm{~h}$ with 1-MA.

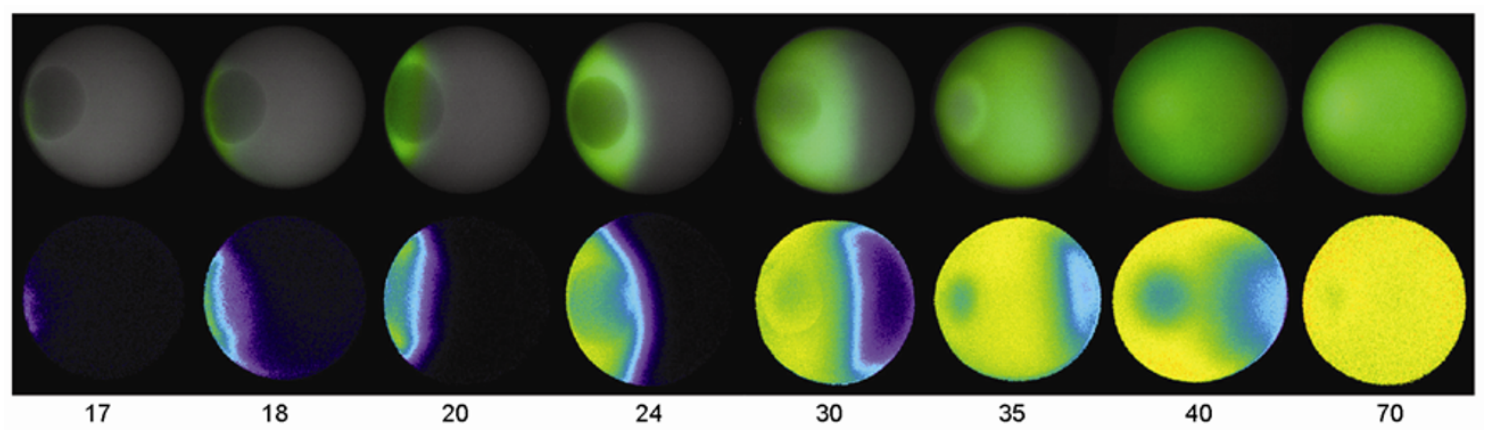

Figure 4 Spatiotemporal pattern of the $\mathrm{InsP}_{3}$-induced $\mathrm{Ca}^{2+}$ increase in the maturing oocytes. Fluorescence and relative fluorescence images of the $\mathrm{Ca}^{2+}$ increase induced by the uncaging of $\mathrm{InsP}_{3}$ in oocytes that had been exposed to 1-MA for differential time. The animal pole of the oocytes containing the nucleus is much more sensitive to InsP $\mathrm{P}_{3}$ than the rest of the cell. During maturation, the increased sensitivity of the $\mathrm{Ca}^{2+}$ stores to InsP $\mathrm{P}_{3}$ spreads to the vegetal pole. $30 \mathrm{~min}$ after 1-MA treatment, $\mathrm{Ca}^{2+}$ is released more homogenouly throughout the oocyte (minutes after the addition of 1-MA).

egg and spermatozoa at fertilization is also reflected in the electrical responses of the egg. In sea urchin, fertilization potential is preceded by a step-like membrane depolarization of 1-2 $\mathrm{mV}$ in amplitude, presumably due to the acrosome-borne factor that is released near the egg plasma membrane [14]. However, fertilization of starfish $A$. aranciacus does not involve this primary electrical event and the membrane depolarization leads directly to the fertilization potential. The lack of the primary event during the activation of starfish eggs was attributed to the acrosome reaction that takes place remotely at the outer surface of the jelly coat [96]. Hence, the morphological events at fertilization are closely linked to the physiological responses such as $\mathrm{Ca}^{2+}$ signaling and the electrical changes.

\subsection{Roles of NAADP and $\mathrm{InsP}_{3}$ for the $\mathrm{Ca}^{2+}$ response at fertilization}

In echinoderms, the $\mathrm{Ca}^{2+}$ wave at fertilization is usually preceded by a cortical flash which is produced by $\mathrm{Ca}^{2+}$ in flux through voltage-gated ion channels activated by the sperm-induced depolarization of the egg plasma membrane [97] (Figure 5). Our laboratory has shown that NAADP, the most recently added family of $\mathrm{Ca}^{2+}$-mobilizing second messengers, elicits a cortical $\mathrm{Ca}^{2+}$ flash, indicating that NAADP liberates $\mathrm{Ca}^{2+}$ from a pool confined to the vicinity 
of the plasma membrane $[98,99]$. The $\mathrm{Ca}^{2+}$ wave propagated toward the inner cytoplasm and also promoted the elevation of the vitelline envelope as a result of cortical granule exocytosis. At variance with the findings in sea urchin eggs [100], however, the response to NAADP in starfish eggs was not affected by the impairment of lysosomes with bafilomycin A1 or glycyl-L-phenylalanine- $\beta$-naphrylamide (GPN) [99]. Instead, the $\mathrm{Ca}^{2+}$ increase by NAADP was negated by the inhibitor of the L-type $\mathrm{Ca}^{2+}$ channels and required external $\mathrm{Ca}^{2+}$. Hence, in starfish eggs, NAADP appeared to work on $\mathrm{Ca}^{2+}$ channels at the plasma membrane [91]. NAADP also triggered the sperm-evoked initial depolarization (fertilization potential) in starfish eggs by the activation of a $\mathrm{Ca}^{2+}$-mediated inward current, which is mainly carried by $\mathrm{Ca}^{2+}$ and displays an inwardly-rectifying $I-V$ relationship [101,102]. Taken together, these results suggested that NAADP could recapitulate some of the early events induced by the fertilizing sperm.

NAADP and $\mathrm{InsP}_{3}$ might play distinct roles in inducing $\mathrm{Ca}^{2+}$ waves at fertilization. Mature eggs enucleated before the addition of 1-MA still respond to NAADP with $\mathrm{Ca}^{2+}$ signals like in the control eggs. However, the same fragmented egg failed to induce the elevation of the vitelline envelope in response to $\operatorname{InsP}_{3}$ although the amount of liberated $\mathrm{Ca}^{2+}$ was similar to that of the control eggs. Upon fertilization, the enucleated eggs produced comparable $\mathrm{Ca}^{2+}$ signals at the cortex, but the speed of the $\mathrm{Ca}^{2+}$ wave was greatly reduced $(>50 \%)$ in comparison to the control eggs. These results suggested that in starfish NAADP might help trigger the $\mathrm{Ca}^{2+}$ signal at fertilization, whereas $\operatorname{InsP}_{3} \mathrm{R}$ could be more responsible for the propagation of the cell-wide $\mathrm{Ca}^{2+}$ waves. The possibility of removing the nucleus from immature starfish oocytes to study its effect on the pattern of $\mathrm{Ca}^{2+}$ liberation and on the cortical granule exocytosis at fertilization will offer the possibility of identifying the nuclear components responsible for the alteration of the $\mathrm{InsP}_{3}$-sensitive $\mathrm{Ca}^{2+}$ stores in the enucleated mature eggs [103,104].

\subsection{Calcium and actin at fertilization}

The egg cortical actin network is important for $\mathrm{Ca}^{2+}$ signaling and the formation of the fertilization envelope, as well as for the control of monospermy and sperm entry. The classical inhibitor of $\operatorname{InsP}_{3} \mathrm{R}$, heparin, delays and inhibits the propagation of sperm-induced $\mathrm{Ca}^{2+}$ signals, but it also induces drastic hyperpolymerization of the cortical actin subjacent to the plasma membrane [105]. Hence, the inhibitory effect on $\mathrm{Ca}^{2+}$ signaling cannot be solely attributed to its pharmacological effect on $\operatorname{InsP}_{3} \mathrm{R}$, but the potential modulation of $\mathrm{Ca}^{2+}$ release by the actin cytoskeleton in the microenvironment should also be considered. As aforementioned, nearly all experimental paradigms that rearranged cortical actin networks resulted in alteration of either amplitude or spatiotemporal dynamics of $\mathrm{Ca}^{2+}$ signals. Interestingly, when the structure of the cortical actin was modified, the starfish eggs were prone to polyspermy as was observed with heparin, jasplakinolide, and sequestration of PIP2 [105,106]. These eggs also failed to undergo a normal elevation of the fertilization envelope although they were contacted by multiple sperm and produced comparable level of $\mathrm{Ca}^{2+}$ signals at fertilization. Interestingly, the polyspermic eggs induced by heparin displayed morphologically abnormal fertilization cones that eventually failed to engulf the engaged sperm. Since fertilization cone is filled with actin filaments that are continuous to the subjacent regions, the failed block to polyspermy and the aborted sperm entry in these eggs might be attributed to the severely altered actin networks at the subplasmalemmal region and inside the fertilization cones. These results, at variance with previous suggestions [107], add more weight to the idea that a proper structural organization and control of the actin cytoskeleton in the cortex is required for the initiation of the $\mathrm{Ca}^{2+}$ signal and for the cortical granules exocytosis $[86,105]$.

According to the prevailing view, a progressive release of $\mathrm{Ca}^{2+}$ at fertilization arises from the ER initiated by the production of $\mathrm{InsP}_{3}[108,109]$. However, direct measurements of cGMP, cADPr and $\mathrm{InsP}_{3}$ at fertilization have shown that the increase in the level of cGMP is followed by those of cADPr and $\mathrm{InsP}_{3}$, and the major rise of $\mathrm{InsP}_{3}$ takes place only after the $\mathrm{Ca}^{2+}$ wave has already initiated [110]. Likewise, in the experiment where the real time changes of the PIP2 levels at the plasma membrane were monitored in a living cell by the use of the RFP-fused marker that contained the PH domain of PLC- $\delta 1$ RFP, the starfish eggs at fertilization displayed the initial $\mathrm{Ca}^{2+}$ rise nearly $10 \mathrm{sec} b e-$ fore the plasma membrane PIP2 level began to decline as a result of PLC activation [106]. Hence, these results cast a doubt on the role of $\operatorname{InsP}_{3}$ as the very first triggering message of $\mathrm{Ca}^{2+}$ signaling at fertilization.

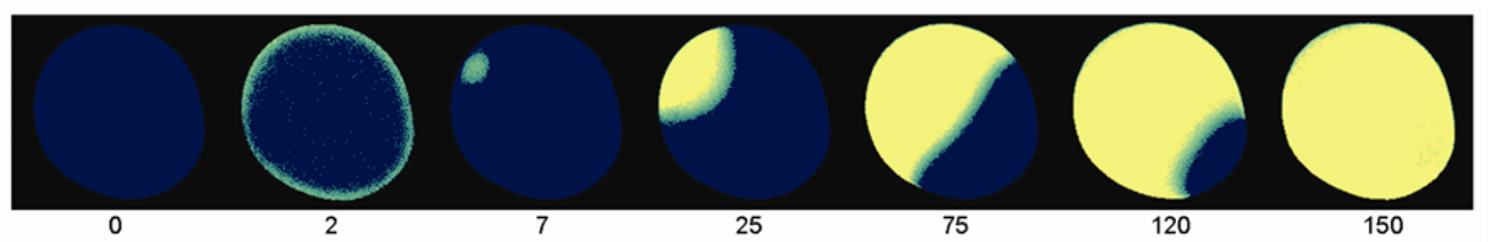

Figure 5 Intracellular $\mathrm{Ca}^{2+}$ release in the mature eggs of starfish at fertilization. Relative fluorescence images of the $\mathrm{Ca}^{2+}$ increase induced by the sperm. The simultaneous release of $\mathrm{Ca}^{2+}$ in the cortex is followed by the $\mathrm{Ca}^{2+}$ wave that starts to propagate from the point of sperm-egg interaction (seconds after sperm-egg interaction). 
The level of plasma membrane PIP2 eventually increases 1-5 min after fertilization of starfish eggs [106], which is in line with the findings in mouse eggs [111]. This late increase of PIP2 at the plasma membrane of the fertilized eggs of starfish is spatiotemporally linked to the formation of numerous spikes that traverse the perivitelline space, which is thought to aid the elevation of the fertilization envelope and the accommodation of the increased lipid surface as the cortical granules are being fused with the plasma membrane during exocytosis [106]. Since many actin-binding proteins have a stretch of basic amino acid residues that interact with the negatively charged head group of PIP2 at the plasma membrane, sequestration of PIP2 could be used as a method to shift the actin dynamics in cells (reviewed in [112-114]). As expected, the starfish mature eggs microinjected with the saturating dose of RFP-PH manifested delayed $\mathrm{Ca}^{2+}$ kinetics and compromised elevation of the fertilization envelope, and the increased tendency to polyspermy [106].

\section{Conclusion}

In nearly all animal species tested, fertilization is accompanied by the rapid rise of intracellular $\mathrm{Ca}^{2+}$ in the eggs. This phenomenon exemplifies one of the most intense $\mathrm{Ca}^{2+}$ increase inside the cell, and the identification of the exact signaling pathways that lead to the mobilization of the $\mathrm{Ca}^{2+}$ wave has been the subject of equally intense research. However, many aspects of the molecular mechanism by which the sperm mobilizes intracellular $\mathrm{Ca}^{2+}$ are still largely unknown. Starfish eggs have provided an excellent model system for the dissection of the $\mathrm{Ca}^{2+}$ response at fertilization. The advantage of the long acrosomal process of the sperm and the possibility to use enucleated oocytes in which the maturation process had been perturbed has allowed us to monitor the $\mathrm{Ca}^{2+}$ signals and the sperm-egg interaction. The experimental paradigm using starfish eggs has provided useful insights into the specific roles played by NAADP and $\mathrm{InsP}_{3}$ at fertilization. Our recent results on the role of the actin cytoskeleton in the modulation of nearly all the early events associated with fertilization have opened a stimulating avenue of research. It will be interesting now to explore the mechanism by which the alteration of the structural organization of the actin cytoskeleton regulates the kinetics and the amount of $\mathrm{Ca}^{2+}$ liberation. The actin cytoskeleton is a dynamic structure that undergoes constant remodeling, and the process is dramatically accelerated by the action of maturation hormone, and even more fiercely by the fertilizing sperm. Within few minutes after fertilization, the egg subplasmalemmal actin fibers rapidly migrate toward the inner cytoplasm. Whether this process is closely linked to the fine regulation of sperm entry would be an interesting topic for future study, especially with the aid of high speed imaging technology. It is also noteworthy that the oscillating cycle of actin polymerization and depolymerization could serve as an additional mechanism to buffer and re- lease $\mathrm{Ca}^{2+}$ in the local environment [115,116]. High level of $\mathrm{Ca}^{2+}$ in the microdomain could be absorbed by polymerizing actin, which itself is a $\mathrm{Ca}^{2+}$-binding protein. Once bound and incorporated into F-actin, the $\mathrm{Ca}^{2+}$ ion is nearly inaccessible to $\mathrm{Mg}^{2+}$ for exchange, and thereby the F-actin could serve as a $\mathrm{Ca}^{2+}$ reservoir. When these actin filaments are depolymerized, $\mathrm{Ca}^{2+}$ would then be released to the local environment, aiding as a supplemental system for $\mathrm{Ca}^{2+}$ release. Whether such a theoretical scenario is actually at work in a living cell is a matter of speculation by now. However, it is obvious that actin is closely linked to the $\mathrm{Ca}^{2+}$-involving cell activities such as fertilization. Like tubulin, which plays a pivotal role during cell division, the amino acid sequence of actin is more than $95 \%$ conserved in all eukaryotic cells. Hence, actin is a highly fundamental eukaryotic invention, and it is not surprising that it has such a comprehensive role at fertilization.

The authors are grateful to R. Graziano and F. Iamunno for their technical assistance with transmission electron microscopy at the SZN.

1 Stricker S A. Comparative biology of calcium signaling during fertilization and egg activation in animals. Dev Biol, 1999, 211: 157-176

2 Santella L, Lim D, Moccia F. Calcium and fertilization: the beginning of life. Trends Biochem Sci, 2004, 29: 400-408

3 Whitaker M. Calcium at fertilization and in early development. Physiol Rev, 2006, 86: 25-88

4 SeGall G K, Lennarz W J. Jelly coat and induction of the acrosome reaction in echinoid sperm. Dev Biol, 1981, 86: 87-93

5 Vacquier V D, Moy G W. Isolation of bindin: the protein responsible for adhesion of sperm to sea urchin eggs. Proc Natl Acad Sci USA, 1977, 74: 2456-2460

6 Collins F, Epel D. The role of calcium ions in the acrosome reaction of sea urchin sperm: regulation of exocytosis. Exp Cell Res, 1977, 106: $211-222$

7 Takahashi Y M, Sugiyama M. Relation between the acrosome reaction and fertilization in the sea urchin. I. Fertilization in Ca-free sea water with egg water treated spermatozoa. Dev Growth Differ, 1973, 15: 261-267

8 Dan J C, Kitahara A, Kohri T. Studies on the acrosome. II acrosome reaction in starfish spermatozoa. Biol Bull, 1954, 107: 203-218

9 Schackmann R W, Shapiro B M. A partial sequence of ionic changes associated with the acrosome reaction of Strongylocentrotus purpuratus. Dev Biol, 1981, 81: 145-154

10 Cantino M E, Schackmann R W, Johnson D E. Changes in subcellular elemental distributions accompanying the acrosome reaction in sea urchin sperm. J Exp Zool, 1983, 226: 255-268

11 Uno Y, Hoshi M. Separation of the sperm agglutinin and the acrosome reaction-inducing substance in egg jelly of starfish. Science, 1978, 200: 58-59

12 Matsumoto M, Kawase O, Islam M S, et al. Regulation of the starfish sperm acrosome reaction by cGMP, $\mathrm{pH}$, cAMP and $\mathrm{Ca}^{2+}$. Int $\mathrm{J}$ Dev Biol, 2008, 52: 523-526

13 Jaffe L A. Fast block to polyspermy in sea urchin eggs is electrically mediated. Nature, 1976, 261: 68-71

14 Dale B, De Felice L J, Taglietti V. Membrane noise and conductance increase during single spermatozoon-egg interactions. Nature, 1978, 275: 217-219

15 Hinkley R E, Wright B D, Lynn J W. Rapid visual detection of sperm-egg fusion using the DNA-specific fluorochrome Hoechst 33342. Dev Biol, 1986, 118: 148-154

16 McCulloh D H, Chambers E L. Fusion membrane during fertilization. J Gen Physiol, 1992, 99: 137-175

17 Rothschild, Swann M M. The fertilization reaction in the sea-urchin egg; the effect of nicotine. J Exp Biol, 1950, 27: 400-406 
18 Dale B, Monroy A. How is polyspermy prevented? Gamete Res, 1981, 4: 151-169

19 Dale B, DeFelice L. Polyspermy prevention: facts and artifacts? J Assist Reprod Genet, 2010, 28: 199-207

20 Byrd E W Jr, Collins F D. Absence of fast block to polyspermy in eggs of sea urchin Strongylocentrotus purpuratus. Nature, 1975, 257: 675-677

21 Allen R, Hagström B. Interruption of the cortical reaction by heat. Exp Cell Res, 1955, 9: 157-167

22 Dale B, Hagström B, Santella L. Partially fertilized sea urchin eggs: an electrophysiological and morphological study. Dev Growth Differ, 1989, 31: 165-170

23 Dale B, Santella L. Sperm-oocyte interaction in the sea-urchin. J Cell Sci, 1985, 74: 153-167

24 Vacquier V D, Tegner M J, Epel D. Protease activity establishes the block against polyspermy in sea urchin eggs. Nature, 1972, 240: 352-353

25 Schuel H. Functions of egg cortical granules. In: Biology of Fertilization. The fertilization response of the egg. Vol 3. C B Metz \& A Monroy Acad Press, Inc., 1985. 1-31

26 Yazaki I. Immunological analysis of the calcium precipitable protein of sea urchin eggs. I) Hyaline layer substance. Embryologia, 1968,10: 131-141

27 Motomura I. Materials of the fertilization membrane in the eggs of echinoderms. Sci Rep Tohoku Imp Univ, 1941,16: 345-363

28 Hertwig O. Beiträge zur Kenntnis der Buildung, Befruchtung und Teilung des tierischen Eies. Morph Jahrb, 1876 1: 374-452

29 Just E E. The fertilization -reaction in Echinarachnius parma. I. Cortical response of the egg to insemination. Biol Bull, 1919, 36: 1

30 Moser F. Studies on cortical layer response to stimulating agents in the Arbacia eggs. I. Response to insemination. J Exp Zool, 1939, 80: 423-446

31 Just E E. The biology of the cell surface. Philadelphia: P. Blakiston's Son \& Co., Inc., 1939

32 Foerder C A, Shapiro B M. Release of ovoperoxidase from sea urchin eggs hardens the fertilization membrane with tyrosine crosslinks. Proc Natl Acad Sci USA, 1977, 74: 4214-4218

33 Kay E S, Shapiro B M.. The formation of the fertilization membrane of the sea urchin egg. In: Biology of Fertilization. The fertilization response of the egg. Vol 3. C B Metz \& A Monroy Acad Press, Inc., 1985. 45-73

34 Turner P R, Jaffe L A, Fein A. Regulation of cortical vesicle exocytosis in sea urchin eggs by inositol 1,4,5-trisphosphate and GTP-binding protein. J Cell Biol, 1986, 102: 70-76

35 Mohri T, Hamaguchi Y. Analysis of the breakdown of cortical granules in echinoderm eggs by microinjection of second messengers. Cell Struct Funct, 1989, 14: 429-438

36 Crossley I, Whalley T, Whitaker M. Guanosine 5'-thiotriphosphate may stimulate phosphoinositide messenger production in sea urchin eggs by a different route than the fertilizing sperm. Cell Regul, 1991, 2: $121-133$

37 Yim D L, Opresko L K, Wiley H S, et al. Highly polarized EGF receptor tyrosine kinase activity initiates egg activation in Xenopus. Dev Biol, 1994, 162: 41-55

38 Dale B, DeFelice L J, Ehrenstein G. Injection of a soluble sperm fraction into sea-urchin eggs triggers the cortical reaction. Experientia, 1985, 41: 1068-1070

39 Kyozuka K, Deguchi R, Mohri T, et al. Injection of sperm extract mimics spatiotemporal dynamics of $\mathrm{Ca}^{2+}$ responses and progression of meiosis at fertilization of ascidian oocytes. Development, 1998, 125: 4099-4105

40 Dale B. Primary and secondary messengers in the activation of ascidian eggs. Exp Cell Res, 1988, 177: 205-211

41 Whitaker M, Irvine R F. Inositol 1,4,5-trisphosphate microinjection activates sea urchin eggs. Nature, 1984, 312: 636-639

42 Churchill G C, O'Neill J S, Masgrau R, et al. Sperm deliver a new second messenger: NAADP. Curr Biol, 2003, 13: 125-128

43 Parrington J, Swann K, Shevchenko V I, et al. Calcium oscillations in mammalian eggs triggered by a soluble sperm protein. Nature, 1996, 379: 364-368

44 Saunders C M, Larman M G, Parrington J, et al. PLC zeta: a sperm-specific trigger of $\mathrm{Ca}(2+)$ oscillations in eggs and embryo de- velopment. Development, 2002, 129: 3533-3544

45 Créton R, Jaffe L F. Role of calcium influx during the latent period in sea urchin fertilization. Dev Growth Differ, 1995, 37: 703-709

46 Deguchi R. Fertilization causes a single $\mathrm{Ca}^{2+}$ increase that fully depends on $\mathrm{Ca}^{2+}$ influx in oocytes of limpets (Phylum Mollusca, Class Gastropoda). Dev Biol, 2007, 304: 652-663

47 Runft L L, Jaffe L A, Mehlmann L M. Egg activation at fertilization: where it all begins. Dev Biol, 2002, 245: 237-254

48 Miyazaki S. Intracellular calcium oscillations in mammalian eggs at fertilization. J Physiol, 2007, 584: 713-714

49 Ducibella T, Fissore R. The roles of $\mathrm{Ca}^{2+}$, downstream protein kinases, and oscillatory signaling in regulating fertilization and the activation of development. Dev Biol, 2008, 315:257-279

50 Carroll D J, Ramarao C S, Mehlmann L M, et al. Calcium release at fertilization in starfish eggs is mediated by phospholipase Cgamma. J Cell Biol, 1997, 138: 1303-1311

51 Ciapa B, Chiri S. Egg activation: upstream of the fertilization calcium signal. Biol Cell, 2000, 92: 215-233

52 Giusti A F, O'Neill F J, Yamasu K, et al. Function of a sea urchin egg Src family kinase in initiating $\mathrm{Ca}^{2+}$ release at fertilization. Dev Biol, 2003, 256: 367-378

53 Townley I K, Schuyler E, Parker-Gür M, et al. Expression of multiple Src family kinases in sea urchin eggs and their function in $\mathrm{Ca}^{2+}$ release at fertilization. Dev Biol, 2009, 327: 465-477

54 Gardiner D M, Grey R D. Membrane junctions in Xenopus eggs: their distribution suggests a role in calcium regulation. J Cell Biol, 1983, 96: 1159-1163

55 Gilkey J C, Jaffe L F, Ridgway E B, et al. A free calcium wave traverses the activating egg of the medaka, Oryzias latipes. J Cell Biol, 1978,76: 448-466

56 Heilbrunn L V. An Outline of General Physiology. 3rd ed. Philadelphia: W. B. Saunders Co., 1952

57 Mazia D. The release of calcium in Arbacia eggs on fertilization. J Cell Comp Physiol, 1937, 10: 291-304

58 Ridgway E B, Gilkey J C, Jaffe L F. Free calcium increases explosively in activating medaka eggs. Proc Natl Acad Sci USA, 1977, 74: 623-627

59 Steinhardt R A, Epel D. Activation of sea-urchin eggs by a calcium ionophore. Proc Natl Acad Sci USA, 1974, 71: 1915-1919

60 Vacquier V D. The isolation of intact cortical granules from sea urchin eggs: calcium lons trigger granule discharge. Dev Biol, 1975, 43: $62-74$

61 Vacquier V D. Dynamic changes of the egg cortex. Dev Biol, 1981, 84: $1-26$

62 Anderson E. Oocyte differentiation in the sea urchin, Arbacia punctulata, with particular reference to the origin of cortical granules and their participation in the cortical reaction. J Cell Biol, 1968, 37: 514-539

63 Chandler D E, Heuser J. Membrane fusion during secretion: cortical granule exocytosis in sex urchin eggs as studied by quick-freezing and freeze-fracture. J Cell Biol, 1979, 83: 91-108

64 Sardet C, Prodon F, Dumollard R, et al. Structure and function of the egg cortex from oogenesis through fertilization. Dev Biol, 2002, 241: $1-23$

65 Santella L, Puppo A, Chun J T. The role of the actin cytoskeleton in calcium signaling in starfish oocytes. Int J Dev Biol, 2008, 52: 571-584

66 Meijer L, Guerrier P. Maturation and fertilization in starfish oocytes. In Rev Cytol, 1984, 86: 129-196

67 Yamamoto K, Yoneda M. Cytoplasmic cycle in meiotic division of starfish oocytes. Dev Biol, 1983, 96: 166-172

68 Yamamoto K. Germinal vesicle contents are required for the cytoplasmic cycle during meiotic division of starfish oocytes. Dev Biol, 1985, 107: 213-219

69 Dale B, de Santis A, Hoshi M. Membrane response to 1-methyladenine requires the presence of the nucleus. Nature, 1979, 282: 89-90

70 Miyazaki S, Hirai S. Fast polyspermy block and activation potential. Correlated changes during oocyte maturation of a starfish. DevBiol, 1979, 70: 327-340

71 Longo F J, Woerner M, Chiba K, et al. Cortical changes in starfish (Asterina pectinifera) oocytes during 1-methyladenine-induced mat- 
uration and fertilisation/activation. Zygote, 1995, 3: 225-239

72 Schroeder T E, Stricker S A. Morphological changes during maturation of starfish oocytes: surface ultrastructure and cortical actin. Dev Biol, 1983, 98: 373-384

73 Santella L, De Riso L, Gragnaniello G, et al. Cortical granule translocation during maturation of starfish oocytes requires cytoskeletal rearrangement triggered by $\mathrm{InsP}_{3}$-mediated $\mathrm{Ca}^{2+}$ release. Exp Cell Res, 1999, 248: 567-574

74 Heil-Chapdelaine R A, Otto J J. Characterization of changes in F-actin during maturation of starfish oocytes. Dev Biol, 1996, 177: 204-216

75 Chiba K, Longo F J, Kontani K, et al. A periodic network of G protein beta gamma subunit coexisting with cytokeratin filament in starfish oocytes. Dev Biol, 1995, 169: 415-420

76 Stricker S A. Structural reorganizations of the endoplasmic reticulum during egg maturation and fertilization. Semin Cell Dev Biol, 2006, 17: 303-313

77 Jaffe L A, Terasaki M. Structural changes in the endoplasmic reticulum of starfish oocytes during meiotic maturation and fertilization. Dev Biol, 1994,164: 579-587

78 Boulware M J, Marchant J S. IP3 receptor activity is differentially regulated in endoplasmic reticulum subdomains during oocyte maturation. Curr Biol, 2005, 15: 765-770

79 Sun L, Haun S, Jones R C, et al. Kinase-dependent regulation of inositol 1,4,5-trisphosphate dependent $\mathrm{Ca}^{2+}$ release during oocyte maturation. J Biol Chem, 2009, 284: 20184-20196

80 Lee B, Vermassen E, Yoon S Y, et al. Phosphorylation of IP3R1 and the regulation of $\left[\mathrm{Ca}^{2+}\right]$ i responses at fertilization: a role for the MAP kinase pathway. Development , 2006, 133: 4355-4365

81 Santella L. The role of calcium in the cell cycle: facts and hypotheses. Biochem Biophys Res Commun, 1998, 244: 317-324

82 Moreau M, Guerrier P, Doree M, et al. Hormone-induced release of intracellular $\mathrm{Ca}^{2+}$ triggers meiosis in starfish oocytes. Nature, 1978, 272: 251-253

83 Witchel H J, Steinhardt R A. 1-Methyladenine can consistently induce a fura-detectable transient calcium increase which is neither necessary nor sufficient for maturation in oocytes of the starfish Asterina miniata. Dev Biol, 1990, 141: 393-398

84 Santella L, Kyozuka K. Reinitiation of meiosis in starfish oocytes requires an increase in nuclear $\mathrm{Ca}^{2+}$. Biochem Biophys Res Commun, 1994, 203: 674-680

85 Santella L, De Riso L, Gragnaniello G, et al. Separate activation of the cytoplasmic and nuclear calcium pools in maturing starfish oocytes. Biochem Biophys Res Commun, 1998, 252: 1-4

86 Kyozuka K, Chun J T, Puppo A, et al. Actin cytoskeleton modulates calcium signaling during maturation of starfish oocytes. Dev Biol, 2008, 320: 426-435

87 Kyozuka K, Chun J T, Puppo A, et al. Guanine nucleotides in the meiotic maturation of starfish oocytes: regulation of the actin cytoskeleton and of $\mathrm{Ca}(2+)$ signaling. PLoS One, 2009, 4: e6296

88 Nusco G A, Chun J T, Ercolano E, et al. Modulation of calcium signalling by the actin-binding protein cofilin. Biochem. Biophys. Res. Commun, 2006, 348: 109-114

89 Chiba K, Kado R T, Jaffe L A. Development of calcium release mechanisms during starfish oocyte maturation. Dev Biol, 1990, 140: 300-306

90 Nusco G A, Lim D, Sabala P, et al. $\mathrm{Ca}(2+)$ response to cADPr during maturation and fertilization of starfish oocytes. Biochem Biophys Res Commun, 2002, 290: 1015-1021

91 Santella L, Kyozuka K, Genazzani A A, et al. Nicotinic acid adenine dinucleotide phosphate-induced $\mathrm{Ca}(2+)$ release. Interactions among distinct $\mathrm{Ca}(2+)$ mobilizing mechanisms in starfish oocytes. J Biol Chem, 2000, 275: 8301-8306

92 Lim D, Ercolano E, Kyozuka K, et al. The M-phase-promoting factor modulates the sensitivity of the $\mathrm{Ca}^{2+}$ stores to inositol 1,4,5-trisphosphate via the actin cytoskeleton. J Biol Chem, 2003, 278: 42505-42514

93 Machaca $\mathrm{K} . \mathrm{Ca}^{2+}$ signaling differentiation during oocyte maturation. J
Cell Physiol, 2007, 213: 331-340

94 Santella L, Ercolano E, Lim D, et al. Activated M-phase-promoting factor (MPF) is exported from the nucleus of starfish oocytes to increase the sensitivity of the Ins $(1,4,5) \mathrm{P} 3$ receptors. Biochem Soc Trans, 2003, 31: 79-82

95 Lim D, Lange K, Santella L. Activation of oocytes by latrunculin A. FASEB J, 2002, 16: 1050-1056

96 Dale B, Dan-Sohkawa M, De Santis A, et al. Fertilization of the starfish Astropecten aurantiacus. Exp Cell Res, 1981, 132: 505-510

97 Townley I K, Roux M M, Foltz K R. Signal transduction at fertilization: the $\mathrm{Ca}^{2+}$ release pathway in echinoderms and other invertebrate deuterostomes. Semin Cell Dev Biol, 2006, 17: 293-302

98 Santella L. NAADP: a new second messenger comes of age. Mol Interv, 2005, 5: 70-72

99 Santella L, Chun J T. Calcium signaling by cyclic ADP-ribose and NAADP. In: Lane M D , Lennarz W J (eds). Encyclopedia of Biological Chemistry 2nd Edition. 2011. (In press)

100 Churchill G C, Okada Y, Thomas J M, Genazzani A A, Patel S, Galione A (2002). NAADP mobilizes $\mathrm{Ca}(2+)$ from reserve granules, lysosome-related organelles, in sea urchin eggs. Cell, 111(5): 703-708

101 Moccia F, Lim D, Nusco G A, et al. NAADP activates a $\mathrm{Ca}^{2+}$ current that is dependent on F-actin cytoskeleton. FASEB J, 2003, 17: 1907-1909

102 Moccia F, Lim D, Kyozuka K, et al. NAADP triggers the fertilization potential in starfish oocytes. Cell Calcium, 2004, 36: 515-524

103 Lim D, Kyozuka K, Gragnaniello G, et al. NAADP ${ }^{+}$initiates the $\mathrm{Ca}^{2+}$ response during fertilization of starfish oocytes. FASEB J, 2001, 15: 2257-2267

104 Hirohashi N, Harada K, Chiba K. Hormone-induced cortical maturation ensures the slow block to polyspermy and does not couple with meiotic maturation in starfish. Dev Biol, 2008, 318: 194-202

105 Puppo A, Chun J T, Gragnaniello G, et al. Alteration of the cortical actin cytoskeleton deregulates $\mathrm{Ca}^{2+}$ signaling, monospermic fertilization, and sperm entry. PLoS One, 2008, 3: e3588

106 Chun J T, Puppo A, Vasilev F, et al. The biphasic increase of PIP2 in the fertilized eggs of starfish: new roles in actin polymerization and $\mathrm{Ca}^{2+}$ signaling. PLoS One, 2010, 5: e14100

107 Whitaker M J, Baker P F. Calcium-dependent exocytosis in an in vitro secretory granule plasma membrane preparation from sea urchin eggs and the effects of some inhibitors of cytoskeletal function. Proc R Soc Lond B Biol Sci, 1983, 218: 397-413

108 Swann K, Whitaker M.. The part played by inositol trisphosphate and calcium in the propagation of the fertilization wave in sea urchin eggs. J Cell Biol, 1986, 103: 2333-2342

109 Thaler C D, Kuo R C, Patton C, et al. Phosphoinositide metabolism at fertilization of sea urchin eggs measured with a GFP-probe. Dev Growth Differ, 2004, 46: 413-423

110 Kuroda R, Kontani K, Kanda Y, et al. Increase of cGMP cADP-ribose and inositol 1,4,5-trisphosphate preceding $\mathrm{Ca}(2+)$ transients in fertilization of sea urchin eggs. Development, 2001, 128: 4405-4414

111 Halet G, Tunwell R, Balla T, et al. The dynamics of plasma membrane PtdIns(4,5)P(2) at fertilization of mouse eggs. J Cell Sci, 2002, 115: 2139-2149

112 Sechi A S, Wehland J. The actin cytoskeleton and plasma membrane connection: PtdIns(4,5)P(2) influences cytoskeletal protein activity at the plasma membrane. J Cell Sci, 2000, 113: 3685-3695

113 Yin H L, Janmey P A. Phosphoinositide regulation of the actin cytoskeleton. Annu Rev Physiol, 2003, 65: 761-789

114 Saarikangas J, Zhao H, Lappalainen P. Regulation of the actin cytoskeleton-plasma membrane interplay by phosphoinositides. Physiol Rev, 2010, 90: 259-289

115 Lange K. Microvillar Ca++ signaling: a new view of an old problem. J Cell Physiol, 1999, 180: 19-34

116 Lange K, Gartzke J. F-actin-based Ca signaling-a critical comparison with the current concept of Ca signaling. J Cell Physiol, 2006, 209: 270-287

Open Access This article is distributed under the terms of the Creative Commons Attribution License which permits any use, distribution, and reproduction in any medium, provided the original author(s) and source are credited. 